\title{
Facilities at Indian Institute of Astrophysics and New Initiatives
}

\author{
Bhuwan Chandra Bhatt
}

Indian Institute of Astrophysics, II Block, Koramangala, Bangalor 560034, India

\begin{abstract}
The Indian Institute of Astrophysics is a premier national institute of India for the study of and research into topics pertaining to astronomy, astrophysics and related subjects. The Institute's main campus in Bangalore city in southern India houses the main administrative set up, library and computer center, photonics lab and state of art mechanical workshop. IIA has a network of laboratories and observatories located in various places in India, including Kodaikanal (Tamilnadu), Kavalur (Tamilnadu), Gauribidanur (Karnataka), Leh \& Hanle (Jammu \& Kashmir) and Hosakote (Karnataka).
\end{abstract}

\section{Introduction}

The Indian Institute of Astrophysics (IIA) traces its history from the creation of an Observatory by East India Company in Madras in 1786 which was later shifted to Kodaikanal, in the beautiful Palani range of hills in Southern India. Madras Observatory was renamed as as Kodaikanal Solar Observatory, and the observatory is known by this name since 1887 . In continuation of a small scale night time astronomy facility started at Kodaikanal, during 1965, a new observatory was started at Kavalur in Tamilnadu for stellar spectroscopy and photometry. The headquarters of the Kodaikanal Observatory shifted to Banaglore in 1971 with the formation of the Indian Institute of Astrophysics, an autonomous research institute fully financed by the Government of India. The institute augmented its research facilities with new campuses and field stations. A summary of these field stations and activities is provided in Sect. 2.

\section{Field stations and activities}

\subsection{Kodaikanal Solar Observatory (Kodaikanal, Tamilnadu)}

The Kodaikanal Solar Observatory is in operation since 1892 continuously. It's main facilities are described in the following paragraphs.

Solar Tunnel Telescope A 60-cm diameter two-mirror coelostat mounted on a 11-m tower platform directs sunlight via a flat mirror into a 60-m long underground horizontal 'tunnel'. A 38-cm f/90 lens forms a 34-cm diameter solar image at the focal plane. 
Telescopes for full disk imaging The Observatory is also well equipped with a series of solar telescopes for full disc observations of the Sun in $\mathrm{H} \alpha$ and K-filtergrams. Daily observations of the Sun have continued for over 100 years. The historical data obtained on photographic plates are digitized and available to the scientific community via a data archive center at IIA. The twin spectro-heliographs giving 6-cm diameter full disc photographs of the Sun in $\mathrm{K} \alpha$ and $\mathrm{H} \alpha$ spectral lines are in regular use. A $46-\mathrm{cm}$ diameter Foucault sidero-stat feeds light to a $30-\mathrm{cm}$ aperture f/22, Cooke triplet lens. The two prism $\mathrm{K} \alpha$ spectro-heliographs were acquired in 1904 and the $\mathrm{H} \alpha$ diffraction grating spectroheliograph was operational in 1911. Since 1912, prominent pictures over the full limb are also being obtained in $\mathrm{K}$ by blocking the solar disc.

\subsection{Vainu Bappu Observatory (Kavalur, Tamilnadu)}

Prof. M.K. V. Bappu, the founder Director of IIA had set up an observatory for the night time observations at Kavalur located in the Javadi hills of Tamilnadu, to access the celestial objects in both the northern and southern hemispheres. The first telescope was of 38-cm (15-inch) aperture, with which astronomical observations were started in 1968 at Kavalur Observatory. In 1972, a 1-m (40-inch) telescope, made by Carl Zeiss Jena, was installed at Kavalur. Additionally, a 75-cm (30-inch) telescope completely designed and fabricated at the workshops of the Indian Institute of Astrophysics was also installed at the observatory.

2.3-m Vainu Bappu Telescope Vainu Bappu's dream project was the 2.3-m (93-inch) aperture telescope, designed and built within the country. Bappu died in 1982 and could not see the completion of this telescope. In a befitting tribute, the then Prime Minister Shri Rajiv Gandhi, at a function held at Kavalur on 6 January 1986, named the observatory as "Vainu Bappu Observatory" (VBO) and the 2.3$\mathrm{m}$ telescope as "Vainu Bappu Telescope" (VBT). The equatorially mounted horse-shoe-yoke structure of the telescope is ideally suited for low latitudes and permits easy observation near the north celestial pole. This telescope has been operated as a national facility and attracts proposals from all over the country and sometimes from outside India. The 2.3-m VBT was the largest telescope in India until the 3.6-m telescope was set up at Devasthal by ARIES in 2016. At prime focus (f/3.25 with a scale of $27^{\prime \prime} / \mathrm{mm}$ ), the 2.3-m VBT is equipped with an imaging camera with a 3-element Wynne corrector and a high-resolution Echelle spectrograph with a detector $(4096 \times 4096$ pixels TEK CCD) with a pixel size of $12 \mu \mathrm{m}$. The 2.3-m VBT operates at Cassegrain focus (f/13 with a scale of $6.8 / \mathrm{mm}$ ) also with a medium-resolution OptoMetrics Research Spectrograph (OMRS) and a detector $(1024 \times 1024$ pixels TEK CCD) with a pixel size of $24 \mu \mathrm{m}$.

\subsection{Gauribidanur Radio Observatory (Gauribidanur, Karnataka)}

Since 1976, the IIA operates a decameter wave radio telescope (GEETEE) jointly with the Raman Research Institute at Gauribidanur about $100 \mathrm{~km}$ north of Bangalore. The telescope consists of 1000 dipoles arranged in a "T" configuration, with a $1.4 \mathrm{~km}$ East-West arm and a $0.5 \mathrm{~km}$ South arm. It has been engaged in the study of radio waves at $34.5 \mathrm{MHz}$ emanating from the Sun and various other diverse objects in the sky. Since 1997, the Gauribidanur radio heliograph (GRAPH) is working to obtain two dimensional images of the solar corona simultaneously at different frequencies in the range 40-150 MHz. The basic receiving element used is a log-periodic dipole (LPD), and the array consists 384 of them configured as 64 groups. The Gauribidanur Low frequency Solar Spectrograph (GLOSS) is a high resolution radio spectrograph used in conjunction with the GRAPH for obtaining dynamic spectra of the transient emission from the solar corona. Based on the theoretical formulations 
for the response of a correlation interferometer, the Gauribidanur Radio Interferometric Polarimeter (GRIP) probes the coronal magnetic field at various heights above the solar surface.

\subsection{Indian Astronomical Observatory (Hanle-Ladakh, Jammu and Kashmir)}

The astronomical community recognizes Mauna Kea site in Hawaii as the best optical site in the world for putting optical telescopes in terms of best seeing and availability of good numbers of cloud free nights in a year. Lots of telescopes of various sizes have been build there during the past century. There is not a lot of space left there and some local environmental issues have come up. Since the last decades of the second millennium, the world started exploring the Chilean high altitude deserts and during this period various telescopes have come up there. Similar Indian efforts were also started at same time in the high altitude cold deserts in the Ladakh region along the trans-Himalayan region. The Hanle valley is situated at high altitudes of $14000-15000 \mathrm{ft}$ above mean sea level. This site is deemed to be excellent for visible, infrared and sub-millimeter observations throughout the year. Specifically for optical observations, conditions yield about 190 photometric nights per year and approximately 255 spectroscopic nights spread evenly over the year with very low annual precipitation (less than $10 \mathrm{~cm}$ ). In addition, there are low ambient temperatures, low humidity, and low concentration of atmospheric aerosols, low atmospheric water vapour, dark nights and low pollution with a median seeing of less than $1^{\prime \prime}$. The main facilities at the Indian Astronomical Observatory (IAO) are described in the following paragraphs.

2-m Himalayan Chandra Optical and Infrared Telescope The Himalayan Chandra Telescope (HCT) is a 2.01-m (6.5-ft) diameter optical-infrared telescope named after India-born Nobel laureate Subrahmanyam Chandrasekhar. It contains a modified Ritchey-Chretien system with a primary mirror made of ULE ceramic, which is designed to withstand the low temperatures it experiences. The telescope was manufactured by Electo-Optical System Technologies Inc. in Tucson (Arizona, USA). Several science instruments are mounted on the telescope: the Himalaya Faint Object Spectrograph (HFOSC), the TIFR Near Infrared Spectrometer and Imager (TIRSPEC), the Hanle Echelle Spectrograph (HESP), the optical CCD imager, etc. This telescope runs totally on solar power generated with a 60KWP solar power system. The complete HCT system is connected with the Center for Research and Education in Science and Technology in Hoskote (Bangalore) via an Indian communication satellite and is operated remotely from there. This telescope is open to the astronomical community, both national and international, and is in successful operation since 2003. More than 200 research papers based on observations obtained with the HCT have been published in peer review journals.

HAGAR Telescope The High Altitude Gamma-Ray Observatory (HAGAR) was established in 2008 at the base of the IAO in Hanle-Ladakh at an altitude of $14000 \mathrm{ft}$. It consists of an array of 7 telescopes (each with 7 mirrors of 90-cm diameter) and has a total light gathering area of $31 \mathrm{~m}^{2}$. HAGAR was installed by IIA in collaboration with the Tata Institute of Fundamental Research (TIFR, Mumbai). The detection technique employs Cerenkov photons produced in the atmosphere by the particle shower generated by energetic gamma-ray photons. High altitude experiments are closer to the source of Cerenkov photons and help in reducing the energy threshold of detection for a given size of telescope. A 21-m diameter Major Atmospheric Cherenkov Experiment (MACE) is being installed by the Bhabha Atomic Research Centre (BARC, Mumbai) for similar experiments. 


\subsection{Center for Research and Education in Science and Technology (Hoskote, Karnataka)}

The Center for Research and Education in Science and Technology (CREST) is situated $35 \mathrm{~km}$ to the northeast of Bangalore near the town Hoskote. The Center houses the control room for the remote operations of the 2-m HCT at the IAO. Astronomers can move the telescope, select the objects and instrument for data acquisition, and collect data from this campus. The data archiving system is set up at this center. This campus houses the M.G.K. Menon Space Science Laboratory and a small laser research facility. A major facility for mirror segments of the Thirty Meter Telescope (TMT) is under construction at this center.

\subsection{Ultra-Violet Imaging Telescope on AstroSat}

AstroSat is India's first dedicated astronomy satellite, launched on 28 September 2015 with the PSLVC30 rocket of the Indian Space Research Organisation (ISRO). The Ultraviolet Imaging Telescope (UVIT), twin 40-cm telescopes covering far-UV to optical bands, is an onboard instrument of AstroSat jointly developed by IIA and the Inter-University Centre for Astronomy and Astrophysics (IUCAA; Pune). The Canadian Space Agency was also involved in the making of the detector system for the UVIT payload on this satellite. UVIT's complete payload is assembled, calibrated, and tested at the M.G.K. Menon Space laboratory at the CREST campus. The instrument has seen first light on 30 November 2015 and is found to meet the expected performance. The UVIT-Payload Operations Center (UVIT-POC) is set up at IIA. For proposal submission and other details, please visit https://www.issdc.gov.in/astro.html.

\subsection{Variable Emission Line Coronagraph on Aditya-L1}

The major scientific objectives of the Variable Emission Line Coronagraph (VELC) on the Aditya-L1 mission are to investigate the existence of intensity oscillations to address coronal heating, study the dynamics and formation of coronal loops and the temperature structure of the coronal features including the cause and acceleration of Coronal Mass Ejections. IIA leads the collaboration with significant assistance from ISRO in the design and development of the VELC instrument and planned that the satellite will be parked in a halo orbit around the Sun-Earth Lagrangian point L1. This vantage point enables interesting new investigations and enables uninterrupted observation of the Sun for long periods.

\section{Summary}

All the facilities as discussed in Sect. 2 are open to all researchers on a collaboration basis. However, for some of the main facilities, we have a time allocating committee and invite the submission of proposals for observing time in cycles. BINA partners are also encouraged to make use of IIA facilities. For more information, IIA's official website http: //www/iiap. res. in can be visited. 


\section{Acknowledgments}

The author is grateful to Prof. G. C. Anupama for critically going through the manuscript and providing the valuable comments. The author is thankful to the local organising committee for arranging the local hospitality. 